https://helda.helsinki.fi

Chain dynamics and intermolecular association in dilute aqueous solutions of isotactic and atactic Poly(Methacrylic acid) : Effect of $\mathrm{NaCl}$ concentration

\title{
Sitar, Simona
}

2019-06-12

Sitar , S , Aseyev , V , Zagar , E \& Kogej , K 2019 , ' Chain dynamics and intermolecular association in dilute aqueous solutions of isotactic and atactic Poly(Methacrylic acid) : Effect of $\mathrm{NaCl}$ concentration ' , Polymer, vol. 174 , pp. 1-10 . https://doi.org/10.1016/j.polymer.2019.04.044

http://hdl.handle.net/10138/329359

https://doi.org/10.1016/j.polymer.2019.04.044

cc_by_nc_nd

acceptedVersion

Downloaded from Helda, University of Helsinki institutional repository.

This is an electronic reprint of the original article.

This reprint may differ from the original in pagination and typographic detail.

Please cite the original version. 


\title{
Chain Dynamics and Intermolecular Association in Dilute Aqueous Solutions of Isotactic and Atactic Poly(Methacrylic Acid): Effect of $\mathrm{NaCl}$ Concentration
}

\author{
Simona Sitar, ${ }^{a, \#}$ Vladimir Aseyev, ${ }^{b}$ Ema Žagar, ${ }^{c}$ and Ksenija Kogej ${ }^{a *}$

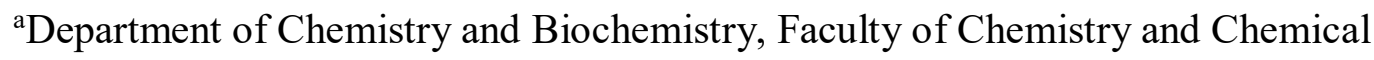 \\ Technology, University of Ljubljana, P.O. Box 537, SI-1000, Ljubljana, Slovenia \\ bepartment of Chemistry, University of Helsinki, P.O. Box 55, FIN-00014 HU, Helsinki, \\ Finland \\ 'National Institute of Chemistry, Hajdrihova 19, 1000 Ljubljana, Slovenia
}

Keywords: poly(methacrylic acid), isotactic, atactic, light scattering, intermolecular association, microgel-like particles, polyelectrolyte mode.

*Corresponding Author:

Ksenija Kogej

Faculty of Chemistry and Chemical Technology,

University of Ljubljana

Večna pot 113

SI-1001 Ljubljana

Slovenia

Tel: +(386-1)-479-8538

E-mail: ksenija.kogej@fkkt.uni-lj.si

\#Present address:

National Institute of Chemistry, Hajdrihova 19, 1001 Ljubljana, Slovenia 


\section{Abstract}

Dynamic and static light scattering measurements were performed on isotactic and atactic forms of poly(methacrylic acid), iPMA and aPMA, respectively, in order to perform a wide survey of their molecular properties and chain dynamics in water as functions of tacticity, degree of neutralization, $\alpha_{\mathrm{N}}$, and salt concentration, $c_{\mathrm{s}}$. The molecular parameters of PMA chains were analyzed at low $\alpha_{\mathrm{N}}$ and chain dynamics (diffusion coefficients and in this connection the polyelectrolyte slow mode behavior) at higher $\alpha_{\mathrm{N}}$. The data revealed that both PMAs form microgel-like aggregates with a core-shell structure at low $\alpha_{\mathrm{N}}(=0$ and 0.25 for aPMA and iPMA, respectively). The distribution of the hydrophilic and hydrophobic functional groups within the aggregates and their compactness depended considerably on chain tacticity and for aPMA also on $c_{\mathrm{s}}$. Further, the effect of $c_{\mathrm{s}}$ on the polyelectrolyte slow diffusion coefficient, $D_{\mathrm{s}}$, of partly $\left(0.25 \leq \alpha_{\mathrm{N}}<1\right)$ or completely charged PMA polyions $\left(\alpha_{\mathrm{N}}=\right.$ 1) was studied. In iPMA solutions, $D_{\mathrm{s}}$ was detected up to $c_{\mathrm{s}}=0.1 \mathrm{M}$, regardless of $\alpha_{\mathrm{N}}$, whereas in aPMA solutions this $c_{\mathrm{s}}$ value gradually decreased with decreasing $\alpha_{\mathrm{N}}$. These differences were also related to tacticity and through that to rigidity of the studied forms of PMA. It is argued that the segregation of uncharged and charged carboxyl groups is operative over the entire $\alpha_{\mathrm{N}}$ region in iPMA solutions. 


\section{Introduction}

Response of polymers in solution to changes in environment is the basis for their use as advanced materials in numerous technological and biochemical applications $[1,2,3]$. In all these cases, polymers respond to certain external stimuli and often self-associate into nanostructured soft materials with a huge variety of properties and potential functionalities. The stimuli to achieve or manipulate polymer self-association (self-assembly) in aqueous solutions are most often changes in $\mathrm{pH}$, ionic strength, temperature, and/or even mechanical stress [4]. Some polymers respond to a combination of two or more stimuli [5], which enables very effective creation of soft materials. Situation is even more complex in case that a polymer chain can acquire charge through ionization of its functional groups, which may lead to additional options for customizing properties, for example through viscosity increase.

Poly(methacrylic acid), PMA, is a synthetic homopolymer that responds to all of the above stimuli and is a potential polyelectrolyte as well. Historically, the reason for the interest in PMA is that its chain exhibits a reversible and cooperative change in conformation as a result of the change in solution's $\mathrm{pH}$, which affects ionization of carboxyl groups [6-10]. In acidic conditions, when unionized, PMA chain is in a compact form, whereas in basic conditions, when fully ionized, it adopts a more extended coil-like conformation, thus enabling the release of ligands bound within the compact PMA core. This principle is widely exploited in various pharmaceutical and biomedical applications [3,11-13].

Much less studied is the fact that behavior of PMA depends significantly on so-called tacticity or stereoregular structure of the polymer chain. Stereoregularity refers to the chain microstructure, i.e. to the spatial distribution of functional groups along the chain, which can be highly regular (isotactic and syndiotactic polymers) or irregular (heterotactic or so-called atactic polymers). The effect of tacticity on properties of polymers is an important issue in polymer chemistry [14]. However, it is surprising that almost no attention is devoted to the 
effect of tacticity on solution behaviour of polyelectrolytes, which is also PMA when its carboxyl groups are ionized. This contribution aims at filling this gap by studying the behavior of isotactic and atactic PMA, iPMA and aPMA, respectively, in the whole range of degrees of neutralization, $\alpha_{N}$, of carboxyl groups, from $\alpha_{N}=0$, when PMA chains are uncharged and can potentially form nanoparticles through self-association, to $\alpha_{N}=1$, when they are fully charged and electrostatic interactions become important. As far as the authors are aware, such a wide survey has not yet been carried out for PMA isomers in relation to chain tacticity and ionic strength of solutions. The ionic strength is normally varied by adding a low molar mass salt. The added salt is important not only when the polymer is highly charged (in electrostatic screening), but also at low charge through the competition between the added ions and the polymer chain for hydration with water molecules. This issue is well known in protein research.

Properties of the highly regular iPMA are relatively poorly investigated, both at low and in particular at gradually increasing charge. Until recently, it was known only that iPMA does not dissolve in water unless around $20 \%$ of its carboxyl groups are ionized (charged), whereas aPMA dissolves already in the pure acid, and that the well-known conformational transition of iPMA takes place along different paths when the polyacid is either protonated or deprotonated [8-13], whereas both paths coincide in the aPMA case [8-10,14-21]. Possible molecular interpretation of these observations was recently offered by a complete thermodynamic analysis of the conformational transition [10]. It was shown therein that iPMA and aPMA respond oppositely to the change in temperature, as clearly proven by the opposite sign of the heat capacity change of the conformational transition. In agreement with these thermodynamic result, van den Bosch et al. [19] have shown by rheology that iPMA at $\alpha_{N} \approx 0.2$ is associated and forms gel upon cooling, whereas Eliassaf and Silberberg [22] demonstrated that the unionized aPMA $\left(\alpha_{N}=0\right)$ does that upon heating. Another striking 
difference is the response of PMA isomers to shear: aPMA chains associate when subjected to shear [23-25], whereas the aggregates between iPMA chains decompose when the solution is sheared and reform in solution at rest [20,21].

These observations apply to the low $\alpha_{\mathrm{N}}$ limit and devote no attention to the role of the added salt, i.e. to the ionic strength of solutions. Moreover, the effect of tacticity on the state of the highly charged PMA polyions (high $\alpha_{N}$ ) is mostly not disclosed, although it is expected that the ionic strength should strongly affect the interactions between polyions themselves and also their interactions with oppositely charged small ions in solution. For example, Jerman et al. [26] have determined the osmotic coefficients of iPMA and aPMA in dependence on degree of ionization, but only in salt free solutions. They concluded that iPMA chain has a higher local charge density than the aPMA one and thus binds counterions more strongly [26,27], which leads to lower osmotic coefficients. The effect of tacticity should also be reflected in diffusion coefficients (chain dynamics) of PMA polyions in solution, which can be determined by dynamic light scattering, DLS. In DLS, strong electrostatic correlations between polyions in low salt conditions lead to a characteristic feature known as the polyelectrolyte, PE, mode. Two relaxation processes are observed by DLS in PE solutions [28-33,3537]. The fast relaxation process stems from a coupled diffusion of polyions and their counterions and the slow relaxation process (revealed as an "apparent" slow diffusion coefficient, $D_{\mathrm{s}}$ ) is attributed to hindered diffusion of polyions due to their high charge. It is labelled by various terms: as an "extraordinary" [28] or even "anomalous" [34] mode, "slow" mode, as "multimacroion-domain" [41] (or "cluster")formation, polyion-lattice-formation [41] etc.

It was realized as early as 1976 in the DLS study of poly(L-lysine) in aqueous $\mathrm{NaBr}$ solutions by Lin et al. [28] that salt concentration, $c_{\mathrm{s}}$, significantly affects Brownian motion of polyions. The authors identified a rather sharp $c_{\mathrm{s}}$ value below which the PE-slow mode 
$\left(D_{\mathrm{s}}\right)$ in PE solutions suddenly appeared and simultaneously light scattering intensity dropped substantially. Sedlák has extensively investigated this feature in PE solutions, including solutions of the usual (or conventional) aPMA form [39-41], and attributed the PE-slow mode to the formation of so-called multimacroion domains in PE solutions with low ionic strength. It was shown that when the concentration of added salt (i.e. the ionic strength) is sufficiently high, the PE-slow mode disappears. We should stress that studies of chain dynamics (slow mode diffusion coefficients) in relation to PMA tacticity are scarce [20,21]. Specifically, there are no reports on the effect of $c_{\mathrm{s}}$ and $\alpha_{\mathrm{N}}$ on $D_{\mathrm{s}}$ of iPMA and aPMA polyions in aqueous solutions.

Consequently, in order to fully understand the behavior of PMA isomers in water, investigations of the effect of ionic strength on the molecular state of PMAs in relation to their tacticity are needed, both at low $\alpha_{\mathrm{N}}$ as well as at higher $\alpha_{\mathrm{N}}$. In this contribution we therefore discuss two subjects: (i) the tendency of iPMA and aPMA towards intermolecular association (low $\alpha_{\mathrm{N}}$ conditions) and of (ii) polyion chain dynamics in dependence on polyion charge (high $\alpha_{\mathrm{N}}$ conditions, i.e. up to $\alpha_{\mathrm{N}}=1$ ) in a broad range of salt $(\mathrm{NaCl})$ concentrations, which served to vary the ionic strength of the solutions. Both, static light scattering, SLS, and DLS were used to determine the size and diffusion properties of polyions. We first investigate the effect of $c_{\mathrm{s}}$ on the hydrodynamic radius, $R_{\mathrm{h}}$, and the radius of gyration, $R_{\mathrm{g}}$, of PMA particles in solution at low $\alpha_{\mathrm{N}}\left(\alpha_{\mathrm{N}}=0\right.$ for aPMA or somewhat higher, i.e. $\alpha_{\mathrm{N}}=0.25$, for iPMA, due to solubility issues) and then treat the effect of $c_{\mathrm{s}}$ on the diffusion coefficients of partly $\left(\alpha_{\mathrm{N}}<1\right)$ or completely charged polyions $\left(\alpha_{\mathrm{N}}=1\right)$. Such a wide survey of tacticity effects on chain dynamics (in particular on so-called polyelectrolyte slow mode) in solutions of iPMA and aPMA is presented for the first time. 


\section{Experimental section}

\subsection{Materials}

PMA samples with well-defined stereoregular composition (tacticity) were from Polymer Source Inc. and were the same as used previously [20,21]. The molecular properties of iPMA were the following: the weight and number average molar mass, $M_{\mathrm{w}}$ and $M_{\mathrm{n}}$, respectively, were $M_{\mathrm{w}}=32000 \mathrm{~g} \mathrm{~mol}^{-1}$ and $M_{\mathrm{n}}=11000 \mathrm{~g} \mathrm{~mol}^{-1}$ and the tacticity was $93 \%$ of isotactic, $3 \%$ of syndiotactic, $4 \%$ of atactic triads. The aPMA sample (Polymer Source Inc., sample no. P14096-MAA: $M_{\mathrm{w}}=189000 \mathrm{~g} \mathrm{~mol}^{-1}$ and $M_{\mathrm{n}}=165000 \mathrm{~g} \mathrm{~mol}^{-1}$ ) was rich in syndiotactic triads (almost 70\% syndiotactic). Additional details on properties and preparation of polymers are reported in our previous publications [20,21]. $\mathrm{NaCl}, \mathrm{NaOH}$ (Fixanal), and $\mathrm{HCl}$ (Fixanal) were from Merck KGaA. Triple distilled water was used to prepare all solutions. All solvents (water and aqueous $\mathrm{NaCl}$ solutions) were filtered through $0.1 \mu \mathrm{m}$ filters prior to solution preparation.

\subsection{Preparation of solutions}

Stock solutions of iPMA and aPMA in water at various $\alpha_{\mathrm{N}}$ values were prepared as reported previously [20] by bearing in mind that iPMA is not soluble in water or in aqueous $\mathrm{NaCl}$ solutions unless $\alpha_{\mathrm{N}}$ exceeds $\sim 0.2$. Thus, solid iPMA was first suspended in water and neutralized to around $\alpha_{\mathrm{N}} \approx 0.5$ by adding $1 \mathrm{M} \mathrm{NaOH}$ so that the polymer dissolved completely. The $\alpha_{\mathrm{N}}$ values below 0.5 were then prepared by adding $1 \mathrm{M} \mathrm{HCl}$ and those above 0.5 by adding $1 \mathrm{M} \mathrm{NaOH}$. Salt $(\mathrm{NaCl})$ concentration, $c_{\mathrm{s}}$, in these stock solutions was adjusted to the desired value by adding a calculated amount of either $0.1 \mathrm{M}\left(c_{\mathrm{s}}<0.07 \mathrm{M}\right)$ or $1 \mathrm{M} \mathrm{NaCl}$ $\left(c_{\mathrm{s}} \geq 0.07 \mathrm{M}\right)$.

Solid aPMA, which is soluble in water at any $\alpha_{\mathrm{N}}$ including $\alpha_{\mathrm{N}}=0$, was dissolved directly in water. The stock solutions with $\alpha_{\mathrm{N}}>0$ were prepared by adding a calculated amount of 1 
$\mathrm{M} \mathrm{NaOH}$ to this solution. As above, required amounts of $0.1 \mathrm{M}$ or $1 \mathrm{M} \mathrm{NaCl}$ were added to stock solutions to adjust $\mathrm{NaCl}$ concentration to the desired value. Experiments for both, iPMA and aPMA, were performed in the range $0.01 \mathrm{M}<c_{\mathrm{s}}<0.5 \mathrm{M}$. Polymer concentration, $c_{\mathrm{p}}$, was set to $c_{\mathrm{p}}=2 \mathrm{~g} \mathrm{~L}^{-1}$ (at $\alpha_{\mathrm{N}}=0$ ), which corresponds to 0.023 moles of COOH groups per volume (abbreviated as $\mathrm{M}$ for mol $\mathrm{COOH} / \mathrm{L}$ ). To make sure that the studied polymer concentration corresponds to dilute region, the overlap concentration, $c_{\mathrm{p}}{ }^{*}\left(=\frac{M_{w}}{N_{A}\left(4 \pi R_{h}^{3} / 3\right)}\right.$, where $N_{\mathrm{A}}$ is the Avogadro constant), was estimated from the measured $R_{\mathrm{h}}$ values of particles (single polymer chains, designated as $R_{\mathrm{h}, 1}$, c.f. Table $\mathrm{S} 1$ in Supporting Information, SI, were used for this estimation). The calculated $c_{\mathrm{p}}{ }^{*}$ was in the range (11-16) and (33-51) $\mathrm{g} \mathrm{L}^{-1}$ for iPMA and aPMA, respectively, showing that measurements were performed well below $c_{\mathrm{p}}{ }^{*}$, i.e. under dilute conditions.

\subsection{Dynamic and Static Light Scattering}

DLS and SLS measurements were conducted with a 3D-DLS spectrometer from LS Instruments $\mathrm{GmbH}$ (Fribourg, Switzerland). The instrument operates in the 3D crosscorrelation mode at a wavelength of incident radiation, $\lambda_{\mathrm{o}}$, equals to $632.8 \mathrm{~nm}$, which is provided by the $20 \mathrm{mV}$ He-Ne laser (Uniphase JDL 1145P). The 3D cross-correlation technique was especially developed for studying strongly scattering samples in order to suppress multiple scattering [42]. Other details regarding the instrumentation were reported previously $[20,21]$ and can be found in SI.

All LS studies were performed at $25{ }^{\circ} \mathrm{C}$. Solutions were carefully filtered through hydrophilic and low protein binding Millex-HV filters (diameter $13 \mathrm{~mm}$, pore size $0.22 \mu \mathrm{m}$ ) directly into the sealable dust-free cylindrical quartz sample cell. The cell was thoroughly purified before filling it with the sample. The samples were allowed to equilibrate for $30 \mathrm{~min}$ 
in the decalin bath before the measurement was initiated. Scattered light was collected in the angular range from $40^{\circ}$ to $150^{\circ}$ with a step of $10^{\circ}$.

Details about the methodological aspects of DLS and SLS can be found in SI and in literature [43-45], so as all other aspects of the data analysis used in this paper [20,21]. Shortly, the correlation functions of the intensity of scattered light, $G_{2}(q, t)$, were recorded simultaneously with the integral time-averaged LS intensities, $I_{\theta} \equiv I_{\mathrm{q}}$, where $q$ (= $\left.\left(4 \pi n_{0} / \lambda_{0}\right) \sin \frac{\theta}{2}\right)$ is the scattering vector, $\theta$ is the angle, and $n_{\mathrm{o}}$ is the refractive index of the medium). The measured intensities were normalized with respect to the Rayleigh ratio, $R$, of toluene. From the measured $R(q)$ for solutions, the radius of gyration, $R_{\mathrm{g}}$, of scattering particles was evaluated (Eqs. S1a and $\mathrm{S} 1 \mathrm{~b}, \mathrm{SI}$ ). In order to determine $R_{\mathrm{g}}$ of particles, two approaches were used: (i) for small particles (with sizes that fulfill the criterion $q R_{\mathrm{g}}<1$ ) the Zimm function (Eq. S2) was employed and (ii) for larger particles the Debye-Bueche scattering function (Eq. S3) was found to be the most suitable for $R_{\mathrm{g}}$ determination. For additional comments on these functions and for particle topologies that they describe, see SI and references 46-52. In order to determine the diffusion coefficients, $D$, and hydrodynamic radius, $R_{\mathrm{h}}$, of particles the $G_{2}(q, t)$ function were converted into the time correlation function of the scattered electric field, $g_{1}(q, t)$, by means of the Siegert's relationship (Eq. S4, SI). Most of the $g_{1}(q, t)$ functions for the herein studied systems were bi-modal (see Eqs. S5), having two well-separated peaks in the calculated relaxation times, $\tau$, distributions. The analysis of the bi-modal $g_{1}(q, t)$ functions was performed as reported previously [21] (see SI) and resulted in the relative distribution functions, $A_{1}\left(q, \tau_{1}\right)$ and $A_{2}\left(q, \tau_{2}\right)$, of the corresponding mean relaxation times, $\tau_{1}$ and $\tau_{2}$, for species 1 and 2 (example of such procedure is demonstrated in Figure S1 together with the procedure for splitting the total LS intensity into separate contributions of both populations). When appropriate, the distributions over $\tau$ were converted into size $\left(R_{\mathrm{h}}\right)$ distributions by means of the relationship $\tau^{-1}=D q^{2}$ and the Stoke-Einstein 
equation (Eq. S6). The evaluated fast translational diffusion coefficient, $D_{\mathrm{f}}$, is associated with individual aPMA chains (small particles with $R_{\mathrm{h}, 1}$ ), whereas the slow translational diffusion coefficient, $D_{\mathrm{s}}$, represents intermolecular associates (large particles with $R_{\mathrm{h}, 2}$ ). In other cases, when the polyelectrolyte effect is observed, the fast and slow relaxation rates, $\Gamma_{\mathrm{f}}$ and $\Gamma_{\mathrm{s}}$ (where $\Gamma=\tau^{-1}=D q^{2}$ ), respectively, do not represent translational diffusion. Consequently, we do not use terminology of sizes but speak about slow and fast diffusive processes. Finally, the determined $R_{\mathrm{g}, 2}$ and $R_{\mathrm{h}, 2}$ values for the aggregates are combined to give so-called shape parameter [44], $\rho\left(=\frac{R_{g, 2}}{R_{h, 0}}\right.$, where $R_{\mathrm{h}, 0}$ is the hydrodynamic radius extrapolated to $\theta=0^{\circ}$ or $q=$ $0)$.

\section{$2.4 \mathrm{Dn} / \mathrm{dc}$ measurements}

A Wyatt Optilab rEX differential refractometer $\left(\lambda_{0}=658 \mathrm{~nm}\right)$ was used to determine the refractive index increment, $\mathrm{d} n / \mathrm{d} c$, of aPMA and iPMA solutions. The $\mathrm{d} n / \mathrm{d} c$ values were measured for aPMA at $\alpha_{\mathrm{N}}=0$ and $c_{\mathrm{s}}=0.01,0.02,0.04,0.05,0.07,0.1,0.15,0.2$, and $0.5 \mathrm{M}$ $\mathrm{NaCl}$, and for iPMA at $\alpha_{\mathrm{N}}=0.25$ and $c_{\mathrm{s}}=0.02,0.05,0.07,0.1,0.2$, and $0.5 \mathrm{M} \mathrm{NaCl}$. In these experiments, the polymer concentration was $0.5-4 \mathrm{~g} \mathrm{~L}^{-1}$.

\section{$2.5 \mathrm{pH}$ measurements}

$\mathrm{pH}$ values were measured with a combined glass electrode (Mettler Toledo Inlab) connected to the $\mathrm{pH}$ meter. The electrode was calibrated with borate and phthalate buffers having $\mathrm{pH}=9.180$ and 4.006 at $25^{\circ} \mathrm{C}$, respectively.

\section{Results and discussions}

A natural way to present the results for PMA isomers is to start with the low $\alpha_{\mathrm{N}}$ limit and continue with studies at higher $\alpha_{\mathrm{N}}$ values. The first part is therefore devoted to intermolecular association, which is observed at low polyion charge (low $\alpha_{\mathrm{N}}$ ), and its dependence on $c_{s}$ and 
the second one to chain dynamics and the polyelectrolyte slow mode behavior at higher polyion charge in dependence on both, $\alpha_{N}$ and $c_{s}$.

3.1 Intermolecular association at $\alpha_{N}=0.25$ (iPMA) and $\alpha_{N}=0$ (aPMA) and structural characteristics of the aggregates

LS was first used to determine the $c_{\mathrm{s}}$ dependence of $R_{\mathrm{h}}, R_{\mathrm{g}}$, and $\rho$ values in aqueous iPMA and aPMA solutions at the lowest possible $\alpha_{\mathrm{N}}$, which allowed solubility of both PMAs: aPMA could be studied at $\alpha_{\mathrm{N}}=0$, while for iPMA $\alpha_{\mathrm{N}}$ had to be higher (see Experimental section) and so $\alpha_{\mathrm{N}}=0.25$ was used in this case. The reason why $\alpha_{\mathrm{N}}=0.25$ was not employed for aPMA is that aggregation is no longer observed in aPMA solution with $\alpha_{\mathrm{N}} \geq 0.25$, irrespective of $c_{\mathrm{s}}$ (see plots of LS intensity in Figure S6b: the sharp drop in LS intensity is interpreted as the disappearance of the aggregates; for more detail see discussion below).

Multi exponential CONTIN analysis of $G_{2}(t)$ functions (shown in Figure S2) results in bimodal distributions of the relaxation times $\tau$ (c.f. insets in Figure S2) at all scattering angles and all $c_{\mathrm{s}}$ values. At the same time, the LS intensity from these solutions is very high (c.f. Figure S6), suggesting that large particles are present in solution. The corresponding fast and slow diffusive processes (or modes) are therefore associated with small (individual) PMA chains and with large intermolecular aggregates between chains, respectively. Figure S3 shows the corresponding dependencies of $\Gamma_{\mathrm{f}}$ and $\Gamma_{\mathrm{s}}$ on $q^{2}$ for small and large particles, respectively. Both curves go through the center of coordinates and thus represent a true translational diffusion (i.e. real particles in solution). Consequently, two dynamic modes can be analyzed individually and the corresponding relative amplitudes of both modes and $D$ and $R_{\mathrm{h}}\left(R_{\mathrm{h}, 1}\right.$ and $\left.R_{\mathrm{h}, 2}\right)$ values can be calculated.

The $\Gamma_{\mathrm{f}}$ versus $q^{2}$ dependences (small particles; Figure S3, the upper panels) are all perfectly linear, showing that small particles have narrow size distributions, whereas the $\Gamma_{\mathrm{s}}$ versus $q^{2}$ curves for the aggregates (Figure S3, the lower panels) show positive deviations 
from linearity at higher $q^{2}$, suggesting that aggregates are much more polydisperse in size. The diffusion coefficients for the small and large particles are determined from the initial slopes of these curves and therefrom the $R_{\mathrm{h}, 1}$ and $R_{\mathrm{h}, 2}$ values are calculated. In case of the aggregates, the radius of gyration, designated as $R_{\mathrm{g}, 2}$, can be determined from the angular dependency of the scattered light intensity (for details see SI). On the other hand, individual chains are too small to display any angular dependency and consequently $R_{\mathrm{g}, 1}$ could not be evaluated. The obtained results for both PMAs are summarized in Table S1 and plotted in Fig. 1 in dependence on $c_{\mathrm{s}}$. Note that the $R_{\mathrm{h}, 2}$ data apply to zero angle.

The hydrodynamic radius of the smaller particles $\left(R_{\mathrm{h}, 1} \sim 10 \mathrm{~nm}\right.$ for iPMA and $\sim 11-13 \mathrm{~nm}$ for aPMA), is independent of $c_{\mathrm{s}}$, whereas the hydrodynamic radius of the aggregates, $R_{\mathrm{h}, 2}$, depends on $c_{\mathrm{s}}$ and the data display a distinctively different dependence for iPMA and aPMA (Fig. 1b). In the iPMA $\left(\alpha_{\mathrm{N}}=0.25\right)$ case, $R_{\mathrm{h}, 2}\left(R_{\mathrm{g}, 2}\right)$ gradually increases with increasing $c_{\mathrm{s}}$ from $\sim 100(\sim 65)$ to $\sim 155(\sim 97) \mathrm{nm}$. Obviously, increase in the ionic strength promotes formation of increasingly larger iPMA aggregates, which is observed also as a strong increase in the absolute LS intensity (Fig. 1a). Taking into account that iPMA chains are charged, the growth of the aggregates with increasing $c_{\mathrm{s}}$ is expected and results from the screening effect of added salt. In addition, Fig. $1 \mathrm{c}$ shows $\rho$ as a function of $c_{\mathrm{s}}$. Similarly to $R_{\mathrm{h}, 2}$ and $R_{\mathrm{g}, 2,} \rho$ is practically independent of $c_{\mathrm{s}}$ in the iPMA case $(\rho=0.60-0.63)$.

For aPMA $\left(\alpha_{\mathrm{N}}=0\right)$, on the other hand, a pronounced maximum in the aggregate size is seen at $c_{\mathrm{s}} \approx 0.07 \mathrm{M}$ and more or less constant (or even decreasing) values are observed for $c_{\mathrm{s}}$ $\geq 0.15 \mathrm{M}$, whereas $R_{\mathrm{h}, 2}$ and $R_{\mathrm{g}, 2}$ increase in this $c_{\mathrm{s}}$ range for iPMA. The lowest $R_{\mathrm{h}, 2}(\approx 120$ $\mathrm{nm})$ and $R_{\mathrm{g}, 2}(\approx 85 \mathrm{~nm})$ are found for aPMA at $c_{\mathrm{s}}=0.01 \mathrm{M}$ and the highest ones, $R_{\mathrm{h}, 2}(\approx 310$ $\mathrm{nm})$ and $R_{\mathrm{g}, 2}(\approx 250 \mathrm{~nm})$ at $c_{\mathrm{s}} \approx 0.07 \mathrm{M}$. The same pattern in the $c_{\mathrm{s}}$ dependency applies to $\rho$ values, which are higher for aPMA $(\rho=0.64-0.8)$ in comparison with iPMA solutions and display a clear maximum $(\rho=0.80)$ at $c_{\mathrm{s}} \approx 0.07 \mathrm{M}$. 


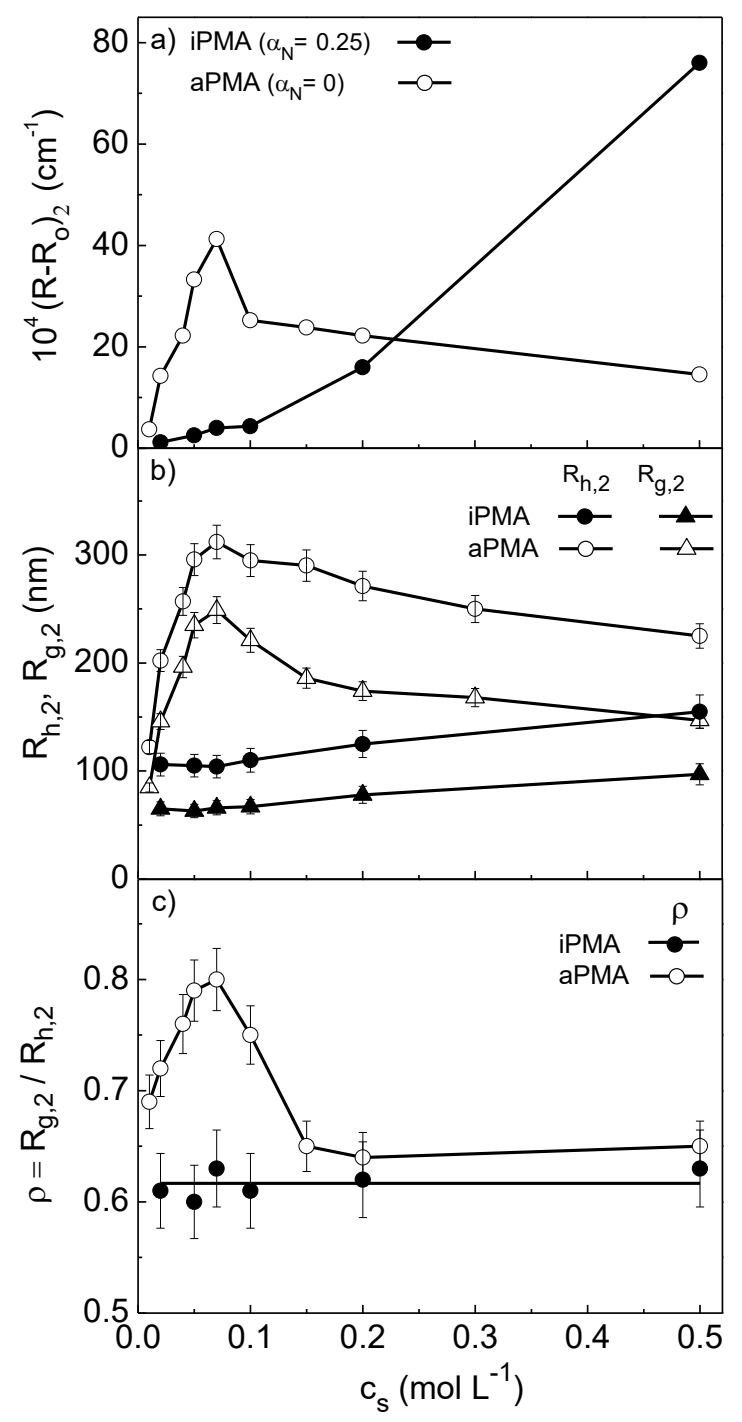

Fig. 1. a) The contribution of the aggregates (index 2) to the total LS intensity (represented as the difference $\left(R-R_{\mathrm{o}}\right)_{2}$, where $R_{\mathrm{o}}$ is the contribution of the solvent to $R$ at $\left.\left.\theta=0\right) ; \mathrm{b}\right)$ the $R_{\mathrm{h}, 2}$ and $R_{\mathrm{g}, 2}$, and c) $\rho\left(=R_{\mathrm{g}, 2} / R_{\mathrm{h}, 2}\right)$ for the aggregates as a function of $c_{\mathrm{s}}$ in aqueous $\operatorname{iPMA}\left(\alpha_{\mathrm{N}}=\right.$ 0.25 ; full symbols $)$ and aPMA $\left(\alpha_{\mathrm{N}}=0\right.$; open symbols $)$ solutions at $25^{\circ} \mathrm{C}$. The contribution of individual chains to the total LS intensity is very small and is not plotted in Fig. 1a. Also, $R_{\mathrm{h}, 1}$ values are not included in the plot because they are small and independent of $c_{\mathrm{s}}$ (for $R_{\mathrm{h}, 1}$ see Table S1). The error bars in Fig. 1a do not exceed the size of the data points.

Parameter $\rho$ is a structure sensitive factor that can be used for qualitative interpretation of the aggregate structure in terms of mass distribution within the aggregates. The $\rho$ values have 
been reported for various particle topologies [43,44,53]. For example, $\rho$ would be $\geq 2$ for fairly stiff and expanded objects (rigid rods), around 1.5 for random coils in a $\theta$ - solvent, 1 for hollow spheres and 0.778 for homogeneous hard spheres. The value of $\rho$ for the herein studied iPMA and aPMA aggregates is considerably lower than 1 (in the range 0.6-0.8, signifying that $R_{\mathrm{h}, 2}$ is much larger than $R_{\mathrm{g}, 2}$ ), which points to structures with a rather high packing density in their interior in comparison to the outer shell/corona. Although $\rho$ for aPMA is close to the value for a homogeneous (impenetrable) hard sphere (e.g. $\rho=0.80$ in $0.07 \mathrm{M} \mathrm{NaCl}$ ), this shape is not anticipated for aPMA aggregates due to the large size of these objects and to the hydrophobic character of the (methyl) side groups on the chain.

Burchard et al. report very low $\rho$ values $(\rho \approx 0.60[54,55]$ or even as low as $\rho \approx 0.35-$ $0.55[56,57])$ for the chemically cross-linked microgel-like particles formed in ethanol solutions of poly-disperse hyper-branched poly(N-vinylimidazole), PVI. In the PVI case, such low $\rho$ is attributed to chains dangling out of the highly contracted core, meaning that mass is concentrated in the center of the particle. An analogous structure is proposed for PMA aggregates in our study. They are similar to microgel-like particles with a core-shell structure, in which the core is considered to be compact and undrained by the solvent, whereas the shell or corona is penetrable for the solvent and thus swollen. In contrast to PVI, PMA chains are not chemically cross-linked but are held within the aggregate by physical forces. These forces are an interplay of rather strong (and in case of iPMA cooperative $[10,19]$; see detailed discussion below) hydrogen bonds, so-called hydrophobic interactions and van der Waals forces.

A question arises why do iPMA $\left(\alpha_{\mathrm{N}}=0.25\right)$ chains form aggregates with a more compact core than do aPMA $\left(\alpha_{\mathrm{N}}=0\right)$ ones, although they are substantially more charged. Intuitively, one would expect a more loose association and therefore higher $\rho$ for iPMA with $\alpha_{\mathrm{N}}=0.25$ due to larger repulsion between charges on the partly ionized chains. However, important is 
first of all chain tacticity and based on this different solute-solvent interactions or affinity of the PMA isomer for the aqueous environment. It has been demonstrated through the analysis of temperature dependence of the thermodynamic functions accompanying ionization of carboxyl groups [10] that iPMA behaves as a typical polar (hydrophilic) and aPMA as a nonpolar (hydrophobic) solute in water. These differences in water affinity can be interpreted by proposing a different distribution of hydrophilic $\left(\mathrm{COOH}\right.$ and also $\left.\mathrm{COO}^{-}\right)$and hydrophobic $\left(\mathrm{CH}_{3}\right)$ groups within the aggregate, which is schematically depicted in Scheme $\mathrm{S} 1$. The aggregates formed between iPMA chains $\left(\alpha_{\mathrm{N}}=0.25\right)$ have most of their ionized $\mathrm{COO}^{-}$ groups situated on the outside of the aggregate and the unionized ones $(\mathrm{COOH})$, together with the hydrophobic $\mathrm{CH}_{3}$ groups, are buried in the aggregate interior. That is, a segregation of $\mathrm{COO}^{-}$and $\mathrm{COOH}$ groups within the aggregate is proposed: its corona consists of charged chain portions extending into the solution, whereas the core is deficient in charges. The iPMA $\left(\alpha_{\mathrm{N}}=0.25\right)$ aggregate is thus actually hydrophilic outwards. This picture agrees with pyrene fluorescence measurements [27]. It has to be noted that normally (for a single isolated chain) an even distribution of $\mathrm{COOH}$ and $\mathrm{COO}^{-}$groups along the polyion chain is imagined, which results in a certain average charge density for the whole chain. On the other hand, by permitting segregation of charged and uncharged groups the local degree of ionization within the core, $\alpha_{N}^{\text {core }}$, can easily be close to 0 , which then allows strong and in case of iPMA also cooperative intermolecular H-bonding between the "core" $\mathrm{COOH}$ groups, as also highlighted in Scheme S1. The basis for this cooperativity is the regular isotactic arrangement of carboxyl groups $[10,19-21]$ that enables a "zipper-like" mechanism of inter-chain connection. That is, when one H-bond is formed the others follow simultaneously, as in cooperative conformational changes. It can be imagined that such an interaction results in a much contracted iPMA aggregate core. 
Along with a lower $\alpha_{\mathrm{N}}$ within the iPMA core, its value in the corona, $\alpha_{N}^{\text {corona }}$, must be higher than 0.25 in order to assure an average $\alpha_{\mathrm{N}}$ of 0.25 . For example, if one quarter (or one third) of the $\mathrm{COOH}$ groups on the iPMA chain is incorporated in the core of the aggregate (with $\alpha_{N}^{\text {core }}=0$ ), then $\alpha_{N}^{\text {corona }}$ for the remaining three quarters (or two thirds) of the chain in the corona, with the residual $\mathrm{COOH}$ and all $\mathrm{COO}^{-}$groups, increases to $\alpha_{N}^{\text {corona }} \approx 0.34$ (or 0.38 ). It is therefore not surprising that the water exposed parts of iPMA chains swell substantially, because ions are usually strongly hydrated. Hydration (swelling) of the corona and simultaneous cooperative H-bonds within the core lead to very low $\rho$ values for the iPMA $\left(\alpha_{\mathrm{N}}=0.25\right)$ aggregates. The described situation (segregation of $\mathrm{COOH}$ and $\mathrm{COO}^{-}$groups within the aggregate) is consistent with the tendency that the concentration of charges on partially ionized polyions is always larger at chain ends, a trend that may even be larger with the isotactic chains.

Similar findings were reported previously for poly(ethacrylic acid), PEA [58], where the strong $\mathrm{H}$-bonding between suitably positioned $\mathrm{COOH}$ groups was even shown to prevent the ionization of the "core" $\mathrm{COOH}$ groups by $\mathrm{NaOH}$. Tao et al. [59] have demonstrated by density functional theory calculations that an electron-donating (or electron-withdrawing) capability of the substituent $\mathrm{R}\left(=\mathrm{CH}_{3}, \mathrm{C}_{2} \mathrm{H}_{5}, \ldots\right)$ bound next to $\mathrm{COOH}$ affects the acidity (and other molecular propertiers, particularly those related to hydrogen bonding) of monomeric carboxylic acids relative to the simplest analog, i.e. formic acid $(\mathrm{H}-\mathrm{COOH})$. The electron-donating groups (such as the hydrophobic $\mathrm{CH}_{3}$ or $\mathrm{C}_{2} \mathrm{H}_{5}$ groups) enhance the electron density at the proton, which results in a shorter $\mathrm{O}-\mathrm{H}$ bond and in lower acidity of the monomeric carboxylic acid, meaning that it is more difficult to detach the proton from $\mathrm{COOH}$. In agreement with the findings for monomeric acids, it was experimentaly observed in case of polymeric carboxylic acids that PMA and PEA ( $\mathrm{R}=\mathrm{CH}_{3}$ and $\mathrm{C}_{2} \mathrm{H}_{5}$, respectively) are weaker acids than poly(acrylic acid), PAA $(\mathrm{R}=\mathrm{H})[6,8-10,16-18,58]$. With polymeric 
carboxylic acids, one has to take into account also the effect of multiple and adjacent acidic sites and the favorable (or unfavorable) microstructure of the chain (tacticity) on the acidity and the strength of eventual intermolecular H-bonds. Van den Bosch et al. [19] have for example argued that intermolecular association between iPMA chains through cooperative Hbonding is very strong and can only be disturbed by solvents (such as DMF and DMSO) capable of forming stronger $\mathrm{H}$-bonds with $\mathrm{COOH}$ groups than water or $\mathrm{COOH}$ themselves. We conclude that this cooperativity in case of iPMA is the key to understanding its complex behavior in aqueous solutions. The interplay of three factors is crucial: (i) highly regular micro-structure of the polymer chain, (ii) the presence of (hydrophobic and electrondonating) $\mathrm{CH}_{3}$ groups bound next to $\mathrm{COOH}$ groups, and (iii) the sequence and proximity of many functional sites.

The distribution of functional groups on the atactic chain is irregular, which prevents the favorable interplay of the above factors. In addition, aPMA $\left(\alpha_{N}=0\right)$ is almost un-charged. The compact interior of the aPMA aggregates at $\alpha_{\mathrm{N}}=0$ is considerably more nonpolar than the interior of the iPMA ones at $\alpha_{\mathrm{N}}=0.25$, as demonstrated by fluorescence measurements [27]. This is a result of more $\mathrm{CH}_{3}$ groups residing in the interior of the aPMA aggregates (Scheme S1), which can relatively easily be incorporated within the core due to their irregular positions on the chain and due to more chain flexibility of the aPMA relative to the iPMA chain. The irregular distribution of $\mathrm{COOH}$ groups on aPMA also decreases the H-bonding strength. The cooperativity is absent and thus the core of the aPMA aggregates is less contracted, whereas the corona is less swollen in comparison with iPMA, as it contains only a few $\mathrm{COO}^{-}$groups. Clearly, we cannot speak about segregation in this case. All this leads to higher $\rho$ values in the aPMA case.

To get an additional confirmation of the proposed microgel-like structure of iPMA and aPMA aggregates, we have carried out a detailed analysis of the angular dependency of light 
scattered by the aggregates. The particle scattering function, $P(q)$, was calculated and is plotted as $\left(q R_{\mathrm{g}}\right)^{2} P(\theta)$ versus $q R_{\mathrm{g}}$ in Fig. 2a (for iPMA at $\alpha_{\mathrm{N}}=0.25$ ) and Fig. 2b (for aPMA at $\alpha_{\mathrm{N}}=0$ ) for some selected $c_{\mathrm{s}}$ values. Solid lines in Figure 2 show the calculated particle scattering functions for some typical particle topologies [60] and points are the experimental data.

The data points in the aPMA $\left(\alpha_{\mathrm{N}}=0\right)$ case extend well above $q R_{\mathrm{g}} \approx 1.5$ (the small particle limit) and agree with the Debye-Bueche scattering function for most $c_{\mathrm{s}}$ values. Only for $c_{\mathrm{s}}=$ 0.07 M, where all size parameters in aPMA solutions display a pronounced maximum (c.f. Fig. 1), the deviation towards the Debye function is observed. The Debye function is generally used to describe the coil conformation, which is penetrable for the solvent. Mass distribution within the aPMA aggregates in solution with $c_{\mathrm{s}}=0.07 \mathrm{M}$ thus resembles that in solvent-drained polymer coils. These results show that the concentration of added $\mathrm{NaCl}$ regulates the compactness of the aggregates between aPMA chains with $\alpha_{\mathrm{N}}=0$.
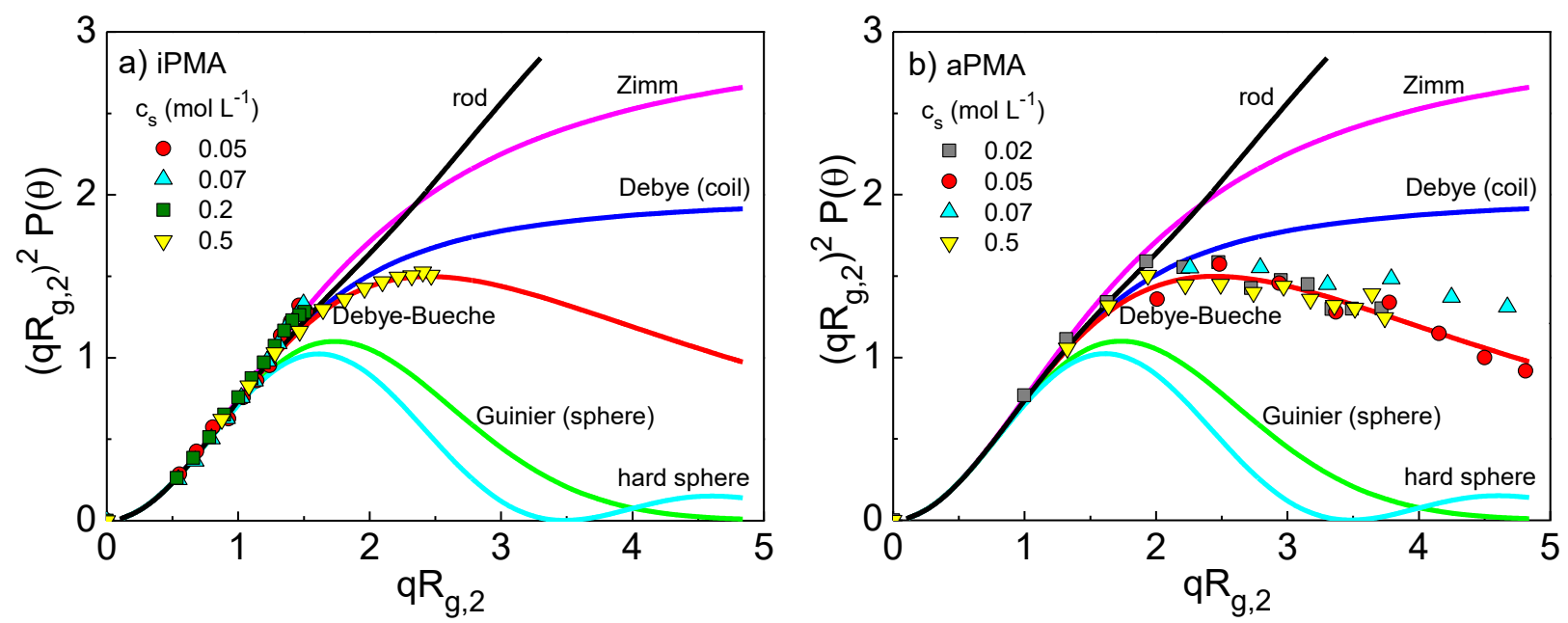

Fig. 2. The dependency of $\left(q R_{\mathrm{g}}\right)^{2} P(\theta)$ on $q R_{\mathrm{g}}$ for six selected particle topologies (solid lines) and the experimental data for intermolecular aggregates (index 2$)$ in a) iPMA $\left(\alpha_{N}=0.25\right)$ and b) aPMA $\left(\alpha_{\mathrm{N}}=0\right)$ solutions at various $\mathrm{c}_{\mathrm{s}}$ values; polymer concentration is $c_{\mathrm{p}}=0.023 \mathrm{M}$. 
An unambiguous classification of the particle topology in iPMA solutions $\left(\alpha_{\mathrm{N}}=0.25\right)$, such as with aPMA, is not so straightforward because the measured data points for most $c_{\mathrm{s}}$ values remain in the region of low $q R_{\mathrm{g}}$ values $\left(q R_{\mathrm{g}}<1.5\right)$ where all topologies (except that for a hard sphere) agree. Only the data series for the highest $c_{\mathrm{s}}(=0.5 \mathrm{M})$ extend to $q R_{\mathrm{g}}$ values close to 3 due to the largest size of the aggregates in this case (see $R_{\mathrm{h}, 2}$ and $R_{\mathrm{g}, 2}$ values in Table S1 and Fig. 1b). These data points clearly fit the Debye-Bueche function, so as those for aPMA for most $c_{\mathrm{s}}$ values (compare Fig. 2a and b). On the basis of these observations and of the low $\rho$ values we conclude that both PMA isomers form similar aggregates in aqueous $\mathrm{NaCl}$ solutions at $T=25^{\circ} \mathrm{C}$ as far as mass distribution is concerned. However, the particular distribution of functional groups is different, as discussed in detail above, and contributes to different degree of compaction on one hand and swelling on the other. As a result, the iPMA and aPMA aggregates differ considerably in the detailed dependence of size parameters on the ionic strength of the medium, regulated by $c_{\mathrm{s}}$. The primary role of added $\mathrm{NaCl}$ is to screen electrostatic repulsions. This is important for iPMA $\left(\alpha_{\mathrm{N}}=0.25\right)$ but not so much for aPMA, since its $\alpha_{\mathrm{N}}$ is 0 . However, the added $\mathrm{Na}^{+}$ions may be able to partly replace hydrogen atoms from the unionized $\mathrm{COOH}$ groups and thus increase the degree of ionization, $\alpha_{\mathrm{i}}$, of aPMA at $\alpha_{\mathrm{N}}=0$. In order to follow the effect of $\mathrm{NaCl}$ concentrations on $\alpha_{\mathrm{i}}$ the $\mathrm{pH}$ of PMA solutions $\left(c_{\mathrm{p}}\right.$ $=0.023 \mathrm{M}$ ) was measured in the studied $c_{s}$ range. From $\mathrm{pH}, \alpha_{\mathrm{i}}$ was calculated using the relationship $\alpha_{i}=\alpha_{N}+\frac{\left[\mathrm{H}^{+}\right]+\left[\mathrm{OH}^{-}\right]}{c_{p}}$ (where $\left[\mathrm{H}^{+}\right]$and $\left[\mathrm{OH}^{-}\right]$are activities of hydronium and hydroxide ions, respectively), which follows from the electro-neutrality condition. The calculated $\alpha_{\mathrm{i}}$ values are plotted in Fig. 3a. Evidentely, the ionization of carboxyl groups in aPMA solutions with $\alpha_{\mathrm{N}}=0$ depends a great deal on $c_{s}$. It increases from around $1.6 \%$ in $0.01 \mathrm{M} \mathrm{NaCl}$ to approximately $3 \%$ in $0.5 \mathrm{M} \mathrm{NaCl}$, i.e. by a factor of almost 2 . On the contrary, $\alpha_{\mathrm{i}}(\cong 0.25)$ is independent of $c_{s}$ in iPMA solutions with $\alpha_{\mathrm{N}}=0.25$. The plot in Fig. 3a shows that the increase of $\alpha_{\mathrm{i}}$ with $c_{s}$ in aPMA $\left(\alpha_{\mathrm{N}}=0\right)$ solutions is steeper for $c_{s}$ values 
below $\approx 0.08 \mathrm{M}$, a limit that agrees surprisingly well with the maximum in the LS parameters $\left(c_{s} \approx 0.07 \mathrm{M} ; c . f\right.$. Fig. 1). Interestingly, the measured $\mathrm{d} n / \mathrm{d} c$ values (c.f. Fig. 3b) also show a distinctive maximum in the same $c_{s}$ range. We suggest that the increase in $\alpha_{\mathrm{i}}$ (decrease in $\mathrm{pH}$ ) of aPMA stems from a partial substitution of hydrogen atoms, which are covalently bound to the aPMA chain, with $\mathrm{Na}^{+}$ions. Through this, the aPMA chains acquire a higher charge which leads to increased repulsion and affects also the hydration state of the polymer chain due to more charged $\mathrm{COO}^{-}$groups. Simultaneously with changed hydration, the contrast between scattering particles and the solvent medium (and thus $\mathrm{d} n / \mathrm{d} c$ values) changes as well.
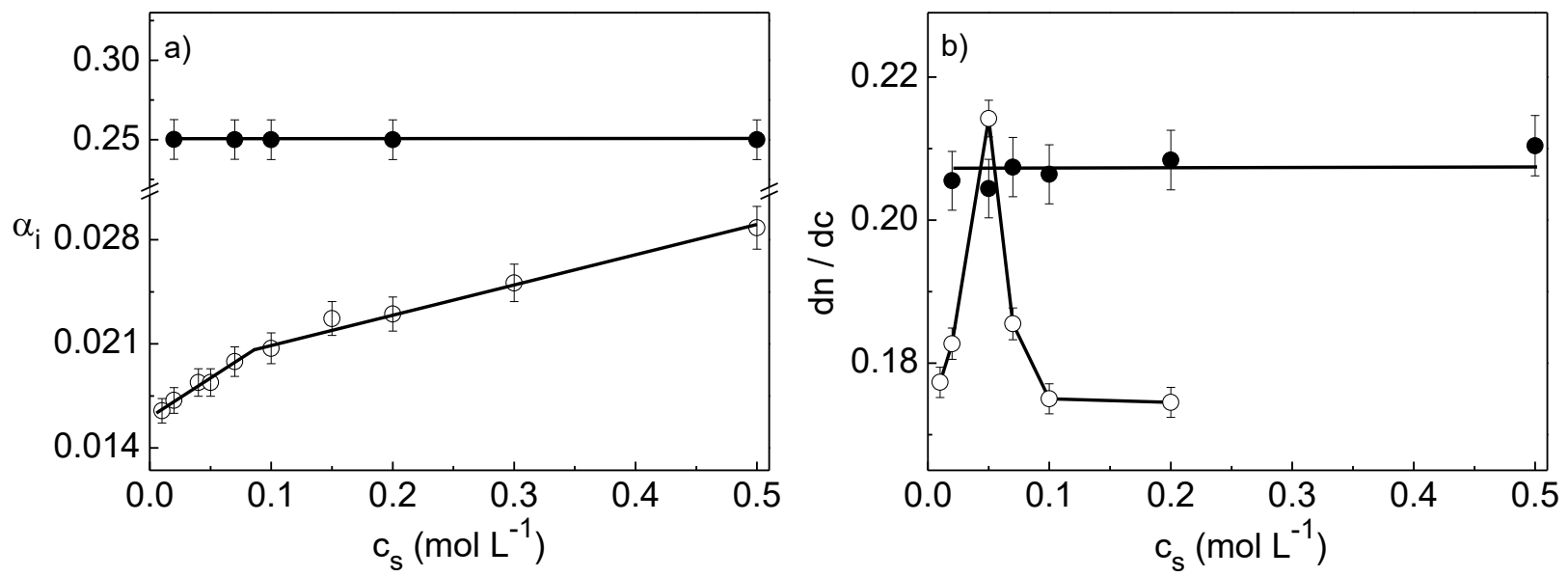

Fig. 3. a) The degree of ionization, $\alpha_{\mathrm{i}}$, and $\left.\mathrm{b}\right)$ the $\mathrm{d} n / \mathrm{d} c$ values in iPMA $\left(\alpha_{\mathrm{N}}=0.25\right.$; full symbols) and aPMA $\left(\alpha_{\mathrm{N}}=0\right.$; open symbols $)$ solutions with $c_{\mathrm{p}}=0.023 \mathrm{~mol} \mathrm{~L}^{-1}$ in dependence on salt concentration, $c_{\mathrm{s}}$.

Because ions are more strongly hydrated than their uncharged counterparts (vis. $\mathrm{COO}^{-}$vs. $\mathrm{COOH})$, more water enters into the physical network of the aPMA aggregates and particles swell, which causes some loosening of the whole structure and results in higher $R_{\mathrm{h}, 2}, R_{\mathrm{g}, 2}$, and also $\rho$ values. For $c_{\mathrm{s}}$ up to $0.07 \mathrm{M}$, the hydration and water uptake work hand in hand. However, at $c_{\mathrm{s}} \approx 0.07 \mathrm{M}$ their delicate balance is destroyed. The screening effect of salt on electrostatic repulsion between $\mathrm{COO}^{-}$groups becomes more efficient, the repulsion decreases 
and the associates can again contract in order to reduce the unfavorable contact of the hydrophobic $\mathrm{CH}_{3}$ groups in the core with water. Simultaneously, the aggregates become less permeable for water, which is observed as a decrease in the size parameters.

On the other hand, the effect of $\mathrm{NaCl}$ on the ionization of $\mathrm{COOH}$ groups on iPMA chains at $\alpha_{\mathrm{N}}=0.25$ is negligible (c.f. Fig. 3a). This is in agreement with previous observations of the effect of other cations (e.g. cationic surfactants) on the ionization of several poly(alkylcarboxylic acids), where it was found that the binding of surfactant cations effects ionization of $\mathrm{COOH}$ groups only for $\alpha_{\mathrm{N}}<0.2$ [61]. We conclude that the delicate effect of $\mathrm{NaCl}$ on intermolecular association plays a role only at $\alpha_{\mathrm{N}}=0$ (for aPMA) and has a negligible effect in the case of partly ionized acid, as is the case with iPMA $\left(\alpha_{\mathrm{N}}=0.25\right)$.

We followed also the effect of the polymer concentration on the aggregate structure of $\operatorname{iPMA}\left(\alpha_{\mathrm{N}}=0.25\right)$ and $\operatorname{aPMA}\left(\alpha_{\mathrm{N}}=0.0\right)$ and arrived at similar conclusions. The data for $c_{\mathrm{p}}=$ 1.5, 2.0, and $2.4 \mathrm{~g} \mathrm{~L}^{-1}$ are collected in Table S2 and Figure S4 (SI). The main conclusion of those results is that the aggregates remain similar to microgel-like particles in the investigated $c_{\mathrm{p}}$ range. The data for aPMA show that the compactness of aggregates increases with increasing $c_{\mathrm{p}}$ ( $\rho$ decreases), whereas with iPMA $c_{\mathrm{p}}$ has no significant effect on $\rho$.

\subsection{Chain dynamics in dependence on $\alpha_{N}$ and $c_{S}$}

In the second part, iPMA and aPMA solutions were studied at higher $\alpha_{\mathrm{N}}$ values $\left(\alpha_{\mathrm{N}} \geq 0.4\right.$ for iPMA and $\alpha_{\mathrm{N}} \geq 0.25$ for aPMA), meaning that polyions are partly or completely $\left(\alpha_{\mathrm{N}}=1\right)$ charged and aggregation is prevented. In this case, $G_{2}(t)$ is used to evaluate diffusion coefficients of polyions in order to study the fast and slow mode behavior of charged PMAs. The focus is on the PE-slow mode, which is a typical feature of highly charged polyions in conditions of low simple salt concentration when their mutual electrostatic interactions are not effectively screened $[32,39,41]$. Examples of the measured $G_{2}(q, t)$ functions for iPMA 
and aPMA solutions with $\alpha_{\mathrm{N}}=1, c_{\mathrm{p}}=0.023 \mathrm{~mol} \mathrm{~L}^{-1}$, and several $c_{\mathrm{s}}$ values are shown in Figure S5 (SI), together with the calculated $\tau$-distributions in the insets.

As noted earlier [31], the LS intensity of salt-free (or low $c_{\mathrm{s}}$ ) PE solutions is low due to the small osmotic compressibility. This is demonstrated for the herein studied iPMA and aPMA solutions in Figure S6 for several $c_{\mathrm{s}}$ values. The total LS intensity drops steeply with increasing polyion charge (increasing $\alpha_{\mathrm{N}}$ ), suggesting that the aggregates between chains disappear for $\alpha_{\mathrm{N}}>0.25$ (iPMA) or $\alpha_{\mathrm{N}}>0$ (aPMA) and only small particles exist in solution (see the $R_{\mathrm{h}}$ data in Tables $\mathrm{S} 3$ and $\mathrm{S} 4$ ). Nevertheless, just like in the low $\alpha_{\mathrm{N}}$ case, the measured correlation functions at the chosen PMA concentration $\left(c_{\mathrm{p}}=0.023 \mathrm{~mol} \mathrm{~L}^{-1}\right)$ show two relaxation modes (see insets in Figure S5), i.e. two diffusion coefficients (two relaxation processes). The higher $D$ value relates to the fast diffusion of single chain, described by $D_{\mathrm{f}}$ (index "f" for fast diffusion) and the lower one to the PE-slow mode feature, described by $D_{\mathrm{s}}$ (index "s" for slow diffusion). The PE-slow mode is not to be mistaken with the slow mode discussed in the previous section, which is related to the slow diffusion of real particles, in the present case to large inter-chain aggregates.

The PE-slow mode at low $c_{\mathrm{s}}$ and high $\alpha_{\mathrm{N}}$ is most clearly detected in $G_{2}(t)$ for aPMA with the highest polyion charge (see for example the case with $c_{\mathrm{s}}=0.02 \mathrm{M}$ and $\alpha_{\mathrm{N}}=1$; Figure $\mathrm{S} 5 \mathrm{~b})$. In the iPMA case, the PE-slow mode is present up to $0.1 \mathrm{M} \mathrm{NaCl}$ for all $\alpha_{\mathrm{N}} \geq 0.4$, whereas for aPMA it is observed up to progressively higher $c_{\mathrm{s}}$ values as $\alpha_{\mathrm{N}}$ increases. This will be in more detail discussed in relation to the slow diffusion coefficients, $D_{\mathrm{s}}$.

Fig. 4 shows some $\Gamma$ vs. $q^{2}$ curves for $\alpha_{\mathrm{N}}=1$ and different $c_{\mathrm{s}}$ values for both PMA isomers. The upper panels apply to the fast diffusion and the lower ones to the PE-slow diffusion mode. Clearly, $\Gamma_{\mathrm{f}}$ is a linear function of $q^{2}$ and goes through the center of coordinates, thus representing true translational diffusion individual polyion chains. The slope of these curves is proportional to $D_{\mathrm{f}}$ and gives their $R_{\mathrm{h}}\left(c . f . R_{\mathrm{h}, 1}\right.$ values in Tables $\mathrm{S} 3$ and $\left.\mathrm{S} 4\right)$. The size $\left(R_{\mathrm{h}, 1}\right)$ of 
single iPMA (aPMA) chains increases from around $9(10) \mathrm{nm}$ at $\alpha_{\mathrm{N}}=0.4(0.25)$ to $11(20)$ $\mathrm{nm}$ for $\alpha_{\mathrm{N}} \geq 0.5$. Higher $R_{\mathrm{h}, 1}$ values for $\alpha_{\mathrm{N}}>0$ are consistent with some chain expansion at higher polyion charge. However, the well-known conformational transition of PMA, which is usually observed in the range $0.2<\alpha_{\mathrm{N}}<0.5$, was not studied in here.
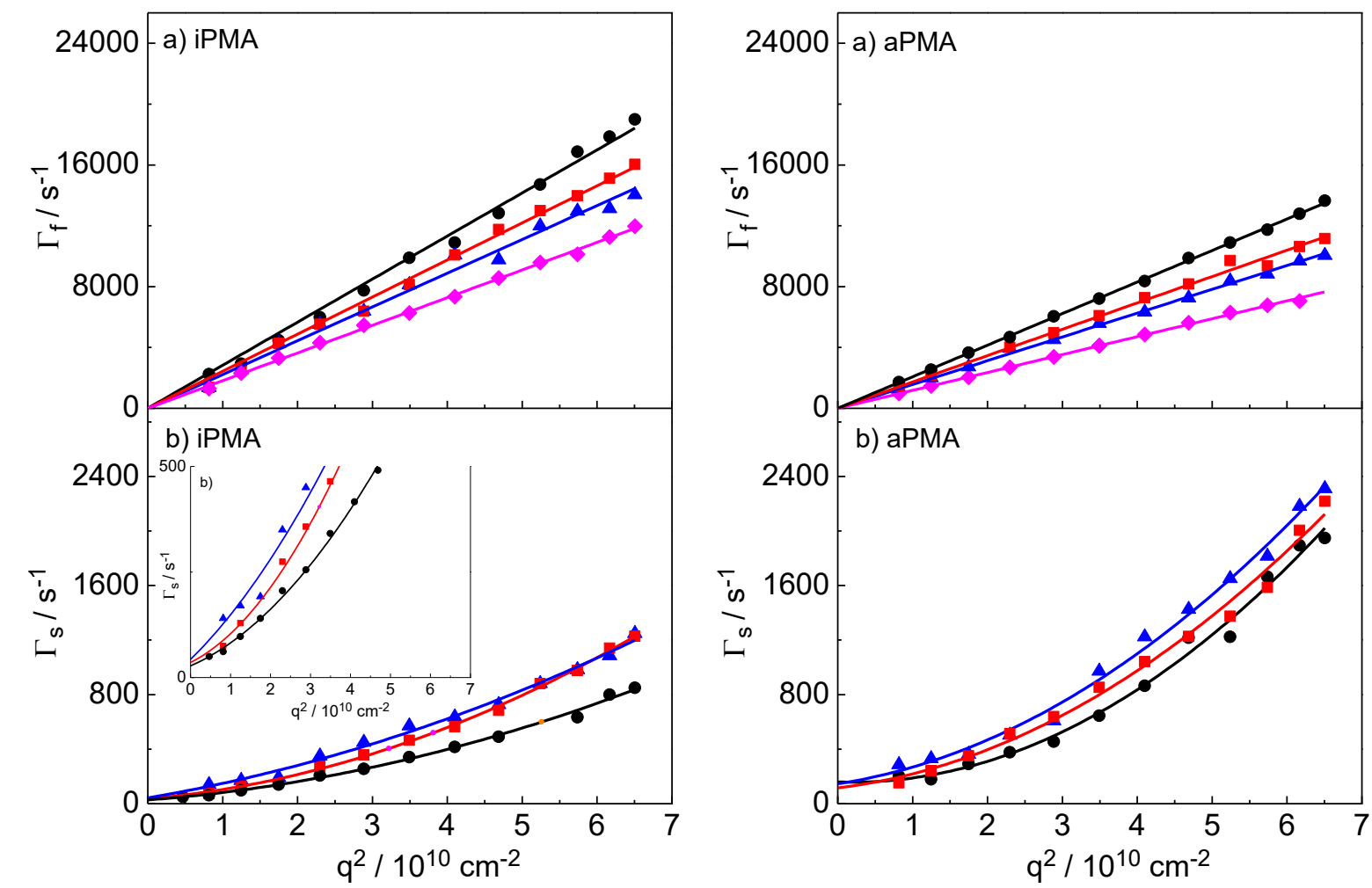

Fig. 4. Decay rates, $\Gamma$, as a function of the square of the scattering vector, $q^{2}$, for $\alpha_{\mathrm{N}}=1$ and various salt concentration: $(\bullet) 0.05,(\boldsymbol{\bullet}) 0.07,(\boldsymbol{\Delta}) 0.1$, and $(\diamond) 0.5 \mathrm{M} \mathrm{NaCl}$; a) fast mode $\left(\Gamma_{\mathrm{f}}\right)$ and b) slow mode $\left(\Gamma_{\mathrm{s}}\right)$. For more details, the $\Gamma_{\mathrm{s}}$ vs. $q^{2}$ curves for iPMA are enlarged in the inset of Fig. $4 b$ (c.f. left lower panel). Note that the slow mode is no longer observed in $0.5 \mathrm{M}$ $\mathrm{NaCl}$, therefore these $\Gamma_{\mathrm{s}}$ vs. $q^{2}$ curves are not presented in Fig. $4 \mathrm{~b}$.

The slow-mode relaxation rate curves $\left(\Gamma_{\mathrm{s}}\right.$ vs $\left.q^{2}\right)$ do not start from the origin of the coordinate system (c.f. lower panels in Fig. 4; compare those with lower panels in Figure S3 for the low $\alpha_{\mathrm{N}}$ case), meaning that this mode is not originating from translational diffusion but owes to the polyelectrolyte effect [45]. This presumption follows also from the fact that 
the total LS intensity is very low (c.f. Figure S6 and the discussion above) and that the angular dependency of LS intensity gives physically meaningless results (not shown). The initial slope of the $\Gamma_{\mathrm{s}}$ vs. $q^{2}$ curves is therefore used simply to evaluate the value of $D_{\mathrm{s}}$.

The combined slow and fast mode behaviors for both PMAs are shown in Fig. 5 as $D$ vs. $c_{\mathrm{s}}$ plots. The $D_{\mathrm{f}}$ values (single chains) span from $\alpha_{\mathrm{N}}=0$ to $\alpha_{\mathrm{N}}=1$, whereas the $D_{\mathrm{s}}$ ones are limited to higher $\alpha_{\mathrm{N}}$, i.e. to the region between $\alpha_{\mathrm{N}}=0.25$ (aPMA) or 0.4 (iPMA) and $\alpha_{\mathrm{N}}=1$, clearly because the slow mode is observed only at sufficiently high polyion charge. The $D_{\mathrm{s}}$ values are more than one order of magnitude lower that the $D_{\mathrm{f}}$ ones. Besides, their dependence on $c_{\mathrm{s}}$ is different. $D_{\mathrm{f}}$ initially steeply decreases with increasing $c_{\mathrm{s}}$ and remains more or less constant for higher $c_{\mathrm{s}}$, with the exception of the aPMA $\left(\alpha_{\mathrm{N}}=0\right)$ case, where it is practically constant (note that in this case, the $D_{\mathrm{s}}$ was not detected). Contrary to $D_{\mathrm{f}}$, the $D_{\mathrm{s}}$ values increase with increasing $c_{\mathrm{s}}$ for both PMAs and disappear at high enough salt concentrations. Interestingly, the $c_{\mathrm{s}}$ limit where this takes place in iPMA solutions is the same for all $\alpha_{\mathrm{N}}$, i.e. $c_{\mathrm{s}}>0.1 \mathrm{M}$, while in aPMA solutions it clearly depends on $\alpha_{\mathrm{N}}$. In aPMA solutions with $\alpha_{\mathrm{N}}=1, D_{\mathrm{s}}$ disappears for $c_{\mathrm{s}}>0.1 \mathrm{M}$, for $\alpha_{\mathrm{N}}=0.75$ and 0.5 the limit is the same $\left(c_{\mathrm{s}}>0.07 \mathrm{M}\right)$ and for $\alpha_{\mathrm{N}}=0.25 D_{\mathrm{s}}$ is no longer detected for $c_{\mathrm{s}}>0.05 \mathrm{M} \mathrm{NaCl}$. Above the quoted $c_{\mathrm{s}}$ values, only the fast diffusion coefficient, $D_{\mathrm{f}}$, is observed. For aPMA, this $c_{\mathrm{s}}$ dependency of $D_{\mathrm{s}}$ on $\alpha_{\mathrm{N}}$ is expected and is due to the lower polyion charge and consequently to weaker electrostatic interactions at lower $\alpha_{\mathrm{N}}$. Based on these results, we suggest that the charge of the aPMA chains changes continuously with $\alpha_{\mathrm{N}}$, i.e. no segregation of ionized and unionized groups is anticipated. By decreasing $\alpha_{\mathrm{N}}$, the overall (effective) charge of the polyion decreases and this leads to weaker electrostatic interactions, which are responsible for the PE-slow mode. 

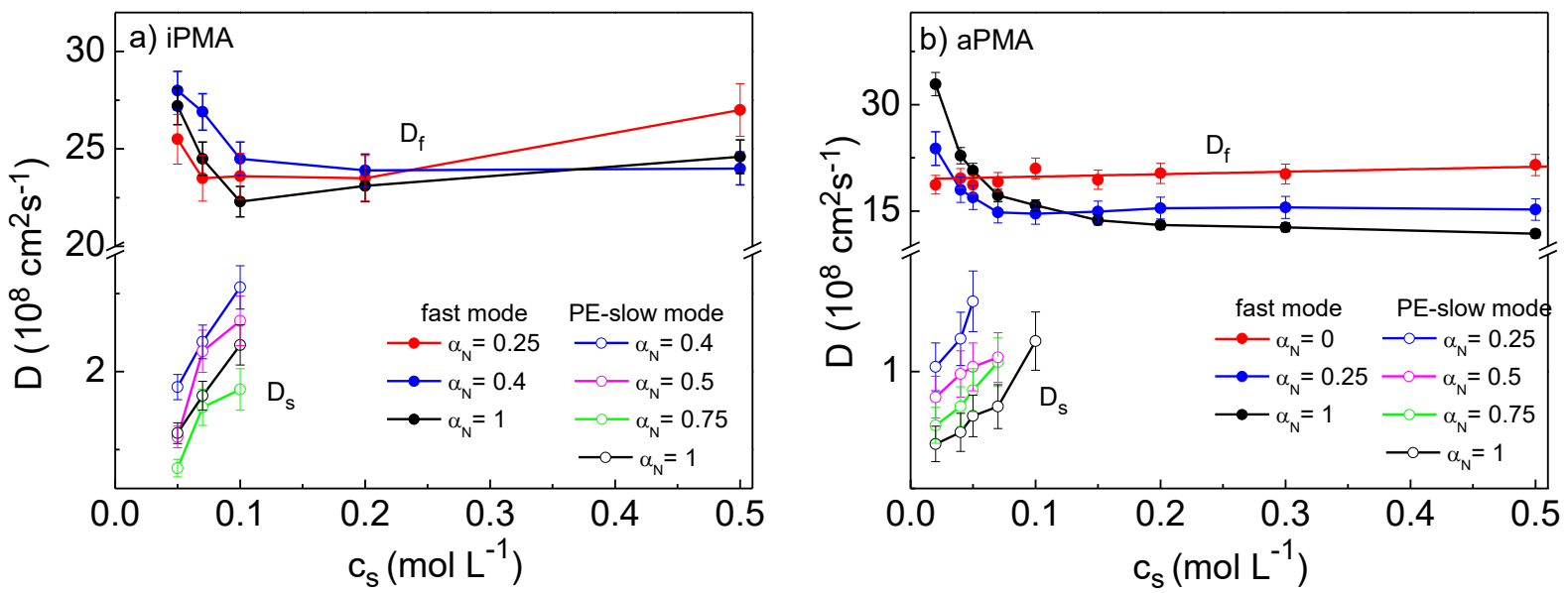

Fig. 5. The slow mode, $D_{\mathrm{s}}$, and fast mode diffusion coefficients, $D_{\mathrm{f}}$, in dependence on $c_{\mathrm{s}}$ for various $\alpha_{\mathrm{N}}$ : a) iPMA and b) aPMA, both with $c_{\mathrm{p}}=0.023 \mathrm{~mol} \mathrm{~L}-1$. In order to clearly present the $D_{\mathrm{s}}$ values, the y-axis contains a brake and is enlarged in the region $D_{\mathrm{s}} \approx(1-3) \times 10^{-8} \mathrm{~cm}^{2}$ $\mathrm{s}^{-1}$.

A question arises why the value of $c_{\mathrm{s}}$, where the PE-slow mode $\left(D_{\mathrm{s}}\right)$ disappears in iPMA solutions, is independent of $\alpha_{\mathrm{N}}$, in obvious contrast to aPMA. We propose that this is because of the isotactic chain configuration and features associate with it, as discussed already above in relation to inter-chain aggregation. Let's propose that the segregation of $\mathrm{COOH}$ and $\mathrm{COO}^{-}$ groups, as proposed above for the low $\alpha_{\mathrm{N}}$ case, persists also at higher $\alpha_{\mathrm{N}}$ where the aggregates have already decomposed. This can be imagined in a way that portions of iPMA chains

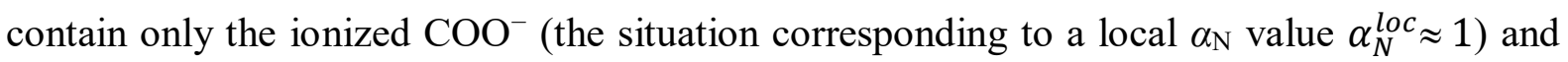
others predominately the unionized $\mathrm{COOH}$ groups (the situation corresponding to $\alpha_{N}^{\text {loc }}=0$ ). As mentioned above, this seems realistic as the concentration of charges is always larger at chain ends; besides, isotacticity may additionally contribute to this tendency. The repulsion between the charged portions of the chains would then be approximately the same, irrespective of their average charge (average $\alpha_{\mathrm{N}}$ value). Because the main factor that leads to the PE-slow mode and the related $D_{\mathrm{s}}$ is the polyion charge, this phenomenon in iPMA 
solutions is detected up to the same $c_{\mathrm{s}}$ value at all $\alpha_{\mathrm{N}}$. This situation resembles the case of cooperative binding of charged surfactants to oppositely polyelectrolytes [61]. The surfactant micelles, which cooperatively form at the polyion, block charges on occupied portions of the chain, but leave the rest of the polyion free and with an unchanged charge density. The charge density is the property that controls various features of PEs, such as counterion binding and also the PE-slow mode (or $D_{\mathrm{s}}$ ), which thus becomes independent of $\alpha_{\mathrm{N}}$.

The dependency of $D_{\mathrm{s}}$ on $\alpha_{\mathrm{N}}$ at a constant $c_{\mathrm{s}}$ value of aPMA solutions shows that $D_{\mathrm{s}}$ increases as the polyion charge $\left(\alpha_{\mathrm{N}}\right)$ decreases. This also makes sense, because a lower charge means weaker electrostatic interactions and less pronounced slow mode. For iPMA, however, the $\alpha_{\mathrm{N}}=0.75$ and 1.0 cases are interchanged: $D_{\mathrm{s}}$ at $\alpha_{\mathrm{N}}=1$ is higher than $D_{\mathrm{s}}$ at $\alpha_{\mathrm{N}}=0.75$. To clarify this observation, more systematic investigations are necessary on the $\alpha_{\mathrm{N}}$ dependency of the PE-slow mode.

\section{Conclusions}

A systematic LS analysis of iPMA and aPMA in aqueous solutions with added $\mathrm{NaCl}$ is performed in order to investigate intermolecular association, limited to low $\alpha_{\mathrm{N}}$ values, and the polyelectrolyte slow mode, observed at high $\alpha_{\mathrm{N}}$ values. These two phenomena are studied in dependence on the ionic strength of the solutions and chain tacticity at a constant polyion concentration $\left(c_{\mathrm{p}}=2 \mathrm{~g} \mathrm{~L}^{-1}\right.$ at $\alpha_{\mathrm{N}}=0$, which corresponds to 0.023 moles of COOH groups per volume). The distinction between the two phenomena, aggregation and PE-slow mode, is made on the basis of (i) the total LS intensity, which drops dramatically upon increasing $\alpha_{\mathrm{N}}$ above 0 (for aPMA) or above 0.25 (for iPMA) as a result of the disappearance of the aggregates, and (ii) physically meaningless angular dependency of the LS intensity of the corresponding PE-slow diffusive mode.

In the case of intermolecular association/aggregation, the geometrical parameters for the small scatterers ( single chains) and for large aggregates are determined for iPMA at $\alpha_{\mathrm{N}}=0.25$ 
and for aPMA at $\alpha_{\mathrm{N}}=0$. While the size of the smaller particles (individual chains) is more or less independent of the ionic strength, the size and shape of the aggregates depends strongly on the salt $(\mathrm{NaCl})$ concentration. For iPMA, the size of the aggregates gradually increases with increasing $c_{\mathrm{s}}$, whereas aPMA shows quite a peculiar dependence of the aggregate size on $c_{\mathrm{s}}$ : the $R_{\mathrm{h}}$ (and $R_{\mathrm{g}}$ ) values initially steeply increases with increasing ionic strength, reach a maximum and thereafter gradually decreases. Thus, the aggregates swell at a particular ionic strength. This result may be relevant for protein solutions, where interaction with water (swelling) controls protein function. From the calculated $\rho$ values, it is concluded that aPMA and iPMA aggregates show characteristics of microgel-like particles with a core-shell structure, in which the core is much denser in comparison with the shell. However, the precise distribution of the polar (hydrophilic) and nonpolar (hydrophobic) functional groups and the associated compactness of the aggregates differs considerably for both isomers. It is suggested that the charged and uncharged carboxyl groups segregate in the iPMA case, which is the basis for very strong and cooperative formation of $\mathrm{H}$-bonds between uncharged chain portions. This is suggested for iPMA in our study for the first time and may be the underlying reason for so-called irreversibility of the conformational transition known for iPMA, which was observed years ago.

Different chemical composition of the aggregate interior (and at the same time aggregate surface) dictates the affinity of PMA isomers for the solvent, i.e. water. Compact core of iPMA is proposed to consist of un-ionized $\mathrm{COOH}$ groups, which are strongly and cooperatively $\mathrm{H}$-bonded, leaving the hydrophobic $\mathrm{CH}_{3}$ groups exposed to water and thus preventing iPMA to dissolve for $\alpha_{\mathrm{N}}<0.2$. In the aPMA case, cooperativity in H-bonding is absent and an opposite distribution of polar and nonpolar groups is proposed: the methyl groups are assembled in the interior of the aggregate and the hydrophilic $\mathrm{COOH}$ groups are 
mostly oriented towards the polar aqueous environment, thus granting solubility of aPMA already at $\alpha_{\mathrm{N}}=0$.

At higher $\alpha_{\mathrm{N}}$ values ( $\alpha_{\mathrm{N}} \geq 0.4$ for iPMA and $\alpha_{\mathrm{N}} \geq 0.25$ for aPMA), where intermolecular association ceases, the electrostatic forces become operative and the PE-slow diffusive mode is detected. In iPMA solutions, the PE-slow mode is present at all $\alpha_{\mathrm{N}} \geq 0.4$ up to $c_{\mathrm{s}}=0.1 \mathrm{M}$, whereas in aPMA solutions this phenomena is most pronounced for the highest polyion charge $\left(\alpha_{\mathrm{N}}=1\right)$, namely up to $0.1 \mathrm{M} \mathrm{NaCl}$, whereas for lower $\alpha_{\mathrm{N}}(\leq 0.75)$ it is seen up to gradually lower $c_{\mathrm{S}}$ values, which is to be expected, the PE-slow mode is to a large extent dependent on the polyion charge: at lower charge (lower $\alpha_{\mathrm{N}}$ ) less salt is needed to suppress electrostatic interactions. In iPMA solutions, on the other hand, the value of $c_{\mathrm{s}}$ where the PEslow mode disappears is surprisingly independent of $\alpha_{\mathrm{N}}$. This remarkable result is again attributed to the isotactic chain configuration. It is presumed that the electrostatic interactions between charged chain parts, which are responsible for the PE-slow mode, may become independent of $c_{\mathrm{s}}$, if the segregation of charged and uncharged carboxyl groups is allowed also at higher $\alpha_{\mathrm{N}}$.

To conclude, isotacticity, which is the reason for the segregation of charged and uncharged carboxyl groups within the iPMA chains, has a far-reaching effect on solution behavior of iPMA in the whole $\alpha_{\mathrm{N}}$ region. The segregation is operative over the entire $\alpha_{\mathrm{N}}$ region and affects both, aggregate formation involving exceptionally strong and cooperative H-bonding between $\mathrm{COOH}$ groups and the polyelectrolyte slow diffusion mode, which is demonstrated by independence of the polyion diffusion coefficients on ionic strength.

\section{Acknowledgments}

This work was financially supported by the Slovenian Research Agency, ARRS, through the Physical Chemistry program P1-0201. In addition, a great part of the experimental work has become possible thanks to the established bilateral agreement between the Academy of 
Finland (Grants no. 132404 and 134581) and the ARRS. The authors are also grateful to the Finnish Society of Sciences and Letters and Magnus Ehrnrooth Foundation for the financial support of the research visits.

\section{Appendix A. Supplementary material}

Detailed aspects of the data analysis, examples of data treatment, and additional experimental results are given in supporting information, SI.

\section{References}

[1] L. Hu, S. Liu, Responsive Polymers for Detection and Sensing Applications: Current Status and Future Developments, Macromolecules 43 (2010) 8315-8330.

[2] T. M. Reineke, Stimuli-Responsive Polymers for Biological Detection and Delivery, ACS Macro Lett. 5 (2016) 14-18.

[3] B. Jeong, A. Gutowska, Lessons from Nature: Stimuli-Responsive Polymers and their Biomedical Applications, Trends Biotechnol. 20 (2002) 305-311.

[4] V. V. Khutoryanskiy, T. K. Georgiou, Temperature-Responsive Polymers: Chemistry, Properties and Applications, Wiley, 2018 pp.145-174.

[5] S. Wiktorowicz, V. Aseyev, H. Tenhu, Chapter 6: Multi-stimuli-responsive Polymers Based on Calix[4]arenes and Dibenzo-18-crown-6-ethers, in: Temperature-Responsive Polymers: Chemistry, Properties and Applications, V. V. Khutoryanskiy and T. K. Georgiou, (Eds.), Wiley, 2018 pp.145-174.

[6] A. Katchalsky, P. Spitnik, Potentiometric Titrations of Poly(Methacrylic Acid), J. Polym. Sci. 2 (1947) 432-446.

[7] A. F. Olea, J. K. Thomas, Fluorescence Studies of the Conformational Changes of Poly(methacrylic acid) with pH, Macromolecules 22 (1989) 1165-1169. 
[8] V. Crescenzi, Some Recent Studies of Polyelectrolyte Solutions, Adv. Polym. Sci. 5 (1968) 358-386.

[9] B. Jerman, K. Kogej, Fluorimetric and Potentiometric Study of the Conformational Transition of Isotactic and Atactic Poly(Methacrylic Acid) in Mixed Solvents, Acta Chim. Slov. 53 (2006) 264-273.

[10] K. Kogej, Thermodynamic Analysis of the Conformational Transition in Aqueous Solutions of Isotactic and Atactic Poly(Methacrylic Acid) and the Hydrophobic Effect, Polymers 8 (2016) 168.

[11] V. V. Khutoryanskiy, Hydrogen-bonded Interpolymer Complexes as Materials for Pharmaceutical Applications, Int. J. Pharm. 334 (2007) 15-26.

[12] Z. Qu, H. Xu, H. Gu, Synthesis and Biomedical Applications of Poly(Methacrylic Acid) Brushes, ACS Appl. Mater. Interfaces 7 (2015) 14537-14551.

[13] A. N. Zelkin, A. D. Price, B. Städler, Poly(Methacrylic Acid) Polymer Hydrogel Capsules: Drug Carriers, Sub-compartmentalized Microreactors, Artificial Organelles, Small 6 (2010) 2201-2207.

[14] K. Hatada, Stereoregular Uniform Polymers, J. Polym. Sci., Part A: Polym. Chem. 37 (1999) 245-260.

[15] E. M. Loebl, J. J. O’Neill, Solution properties of Isotactic Poly(Methacrylic Acid), J. Polym. Sci. 45 (1960) 538-540.

[16] J. C. Leyte, H. M. R. Arbouw-van der Veen, L. H. Zuiderweg, Irreversible Potentiometric Behavior of Isotactic Poly(Methacrylic Acid), J. Phys. Chem. 76 (1972) 25592561.

[17] M. Nagasawa, T. Murase, K. Kondo, Potentiometric Titration of Stereoregular Polyelectrolytes, J. Phys. Chem. 69 (1965) 4005-4012. 
[18] J. C. Leyte, M. Mandel, Potentiometric Behavior of Poly(methacrylic acid), J. Polym. Sci., Part A 2 (1964) 1879-1891.

[19] E. van den Bosch, Q. Keil, G. Filipcsei, H. Berghmans, H. Reynaers, Structure Formation in Isotactic Poly(methacrylic acid), Macromolecules 37 (2004) 9673-9675.

[20] S. Sitar, V. Aseyev, K. Kogej, Differences in Association Behavior of Isotactic and Atactic Poly(Methacrylic Acid), Polymer 55 (2014) 848-854.

[21] S. Sitar, V. Aseyev, K. Kogej, Microgel-like Aggregates of Isotactic and Atactic Poly(Methacrylic Acid) Chains in Aqueous Alkali Chloride Solutions as evidenced by Light Scattering, Soft Matter 10 (2014) 7712-7722.

[22] J. Eliassaf, A. Silberberg, The Gelation of Aqueous Solutions of Poly(Methacrylic Acid), Polymer 3 (1962) 555-564.

[23] J. Eliassaf, A. Silberberg, A. Katchalsky, Negative Thixotropy of Aqueous Solutions of Polymethacrylic Acid, Nature 176 (1955) 1119.

[24] S. Ohoya, S. Hashiya, K. Tsubakiyama, T. Matsuo, Shear-Induced Viscosity Change of Aqueous Poly(Methacrylic Acid) Solution, Polym. J. 32 (2000) 133-139.

[25] C. Robin. C. Lorthioir, C. Amiel, A. Fall, G. Ovarlez, C. Le Coeur, Unexpected Rheological Behavior of Concentrated Poly(Methacrylic Acid) Aqueous Solutions, Macromolecules 50 (2017) 700-710.

[26] B. Jerman, M. Breznik, K. Kogej, S. Paoletti, Osmotic and Volume Properties of Stereoregular Poly(Methacrylic Acids) in Aqueous Solution: Role of Intermolecular Association, J. Phys. Chem. B 111 (2007) 8435-8443.

[27] N. Vlachy, J. Dolenc, B. Jerman, K. Kogej, Influence of Stereoregularity of the Polymer Chain on Interactions with Surfactants: Binding of Cetylpyridinium Chloride by Isotactic and Atactic Poly(Methacrylic Acid), J. Phys. Chem. B 110 (2006) 9061-9071. 
[28] S. C. Lin, W. I. Lee, J. M. Schurr, Brownian Motion of Highly Charged Poly(Llysine). Effect of Salt and Polyion Concentration, Biopolymers 17 (1978) 1041-1064.

[29] R. G. Smits, M. E. Kuil, M. Mandel, Quasi Elastic Light Scattering Study on Solutions of Linear Flexible Polyelectrolytes at Low Ionic Strength, Macromolecules 27 (1994) 55995608.

[30] B. D. Ermi, E. J. Amis, Domain Structures in low Ionic Strength Polyelectrolyte Solutions, Macromolecules 31 (1998) 7378-7384.

[31] R. Cong, E. Temyanko, P. S. Russo, N. Edwin, R. M. Uppu, Dynamics of Poly(styrenesulfonate) Sodium Salt in Aqueous Solution, Macromolecules 39 (2006) 731739.

[32] K. Zhou, J. Li, Y. Lu, G. Zhang, Z. Xie, W. Chi, Re-examination of Dynamics of Polyeletrolytes in Salt-Free Dilute Solutions by Designing and Using a Novel Neutral-Charged-Neutral Reversible Polymer, Macromolecules 42 (2009) 7146-7154.

[33] R. Cong, E. Temyako, P. S. Russo, N. Edwin, R. M. Uppu, Dynamics of Poly(styrenesulfonate) Sodium Salt in Aqueous Solution, Macromolecules 39 (2006) 731 739.

[34] S. Förster, M. Schmidt, M. S. Antonietti, Static and Dynamic Light Scattering by Aqueous Polyelectrolyte Solutions: Effect of Molecular Weight, Charge Density and Added Salt, Polymer 31 (1990) 781-792.

[35] M. Drifford, J. P. Dalbiez, Light Scattering by Dilute Solutions of Salt-Free Polyelectrolytes, J. Phys. Chem. 88 (1984) 5368-5375.

[36] R. S. Koene, M. Mandel, Quasi-Elastic Light Scattering by Polyelectrolyte Solutions without added Salt, Macromolecules 16 (1983) 973-978. 
[37] K. S. Schmitz, M. Lu, N. Singh, D. J. Ramsay, Ordinary Extraordinary PhaseTransition of Poly(lysine) - Comments, Biopolymers, 23 (1984) 1637-1646.

[38] K. S. Schmitz, An introduction to DLS by Macromolecules, Boston:Academic Press, 1990.

[39] M. Sedlak, Č. Konak, P. Štepanek, J. Jakeš, Semidilute Solutions of Poly(Methacrylic Acid) in the Absence of Salt: Dynamic Light Scattering Study, Polymer 28 (1987) 873-879.

[40] M. Sedlák, Domain Structure of Polyelectrolyte Solutions: Is it Real? Macromolecules $26(1993) 1158-1162$.

[41] M. Sedlák, Long-time stability of Multimacroion Domains in Polyelectrolyte Solutions, J. Chem. Phys.116 (2002) 5246-5255.

[42] C. Urban, P. Schurtenberger, Characterization of Turbid Colloidal Suspensions Using Light Scattering Techniques Combined with Cross-Correlation Methods, J. Colloid Interface Sci. 207 (1998) 150-158.

[43] P. Kratochvil, Classical Light Scattering from Polymer Solutions, Elsevier, Amsterdam, 1987.

[44] W. Schärtl, Light Scattering from Polymer Solutions and Nanoparticle Dispersions, Springer Verlag, Berlin Heidelberg, 2007.

[45] W. Brown, Dynamic Light Scattering: The Method and Some Application, Claredon Press, Oxford, 1993.

[46] B. H. Zimm, The Scattering of Light and the Radial Distribution Function of High Polymer Solutions, J. Chem. Phys. 16 (1948) 1093-1099.

[47] B. H. Zimm, Apparatus and Methods for Measurement and Interpretation of the Angular Variation of Light Scattering; Preliminary Results on Polystyrene Solutions, J. Chem. Phys. 16 (1948) 1099-1166. 
[48] A. Guinier, La diffraction des rayons $\mathrm{X}$ aux très petits angles : application à l'étude de phénomènes ultramicroscopiques, Ann. Phys. 12 (1939) 161-237.

[49] A. Guinier, G. Fournet, Small Angle Scattering from X-Rays, Wiley, New York, 1955.

[50] P. Debye, F. Bueche, Scattering by an Inhomogeneous Solid. J. Appl. Phys. 20 (1949) $518-525$.

[51] P. Debye, F. Bueche, Distribution of Segments in a Coiling Polymer Molecule, J. Chem. Phys. 20 (1952) 1337-1338.

[52] A. Ishihara, Behavior of Linear Polymers in Solution, J. Polym. Sci. 8 (1952) 574-576.

[53] W. Burchard, Solution Properties of Branched Macromolecules, Adv. Polym. Sci. 143 (1999) 113-194.

[54] W. Burchard, Angular Dependence of Scattered Light from Hyperbranched Structures in a Good Solvent. A Fractal Approach, Macromolecules 37 (2004) 3841-3849.

[55] G. Savin, W. Burchard, C. Luca, C. Beldie, Global Solution Properties of Poly $(N-$ vinylimidazole) in Ethanol. Macromolecules and Aggregates, Macromolecules 37 (2004) $6565-6575$.

[56] W. Burchard, M. Schmidt, The Diffusion Coefficient of Branched Polyvinylacetates and of Polyvinylacetate Microgels Measured by Quasielastic Light Scattering, Ber. BunsenGes. Phys. Chem. 83 (1979) 388-391.

[57] M. Schmidt, D. Nerger, W. Burchard, Quasi-Elastic Light Scattering from Branched Polymers: 1. Polyvinylacetate and Polyvinylacetate-Microgels prepared by Emulsion Polymerization, Polymer 20 (1979) 582-588.

[58] S. Peljhan, E. Žagar, J. Cerkovnik, K. Kogej, Strong Intermolecular Association between Short Poly(ethacrylic acid) Chains in Aqueous Solutions, J. Phys. Chem. B 113 (2009) 2300-2309. 
[59] L. Tao, J. Han, F. M. Tao, Correlations and Predictions of Carboxylic Acid $\mathrm{p} K \mathrm{a}$ Values Using Intermolecular Structure and Properties of Hydrogen-Bonded Complexes, J. Phys. Chem. A 112 (2008) 775-782.

[60] M. B. Huglin, Light Scattering from Polymer Solutions, Academic Press:New York, 1972.

[61] K. Kogej, Association and Structure Formation in Oppositely Charged PolyelectrolyteSurfactant Mixtures, Adv. Colloid Interface Sci. 158 (2010) 68-83. 